\title{
ON THE POSSIBLE RATE OF GROWTH OF AN ANALYTIC FUNCTION
}

\author{
BY \\ P. W. KETCHUM
}

Introduction( $\left.{ }^{1}\right)$. The present paper deals with a number of diverse topics, ranging from purely topological considerations, through a general theory of possible distributions of values of an analytic function, to more special theorems on simultaneous expansions of an infinity of analytic functions. No unifying principle is presented as an excuse for treating such a variety of subjects; but there is a slight sequence of argument running throughout. The parts of the paper were actually written in reverse order. The initial investigation (Part III) was started as an attempt to generalize, from $n$ to infinity, a known theorem $\left({ }^{2}\right)$ on simultaneous expansions of $n$ analytic functions. This generalization was found to depend on an affirmative answer to the following question on level curves of an analytic function: Given any sequence of points $a_{1}, a_{2}, \cdots$, in the complex plane, which has the point at infinity as its only limit point, does there exist an analytic function with a level curve $C$ such that $C$ contains a distinct branch about each given point which separates that point from all the other points? This was, in turn, made to depend on a certain problem relative to the possible rate of growth of an integral function.

It was shown long ago by Poincaré $\left({ }^{3}\right)$, Borel $\left({ }^{4}\right)$, and others that an integral function may be made to grow arbitrarily fast along the real axis or along other lines or curves extending to infinity. Our problem was to obtain an affirmative answer to the following related question: Does there exist a sequence of regions $S_{1}, S_{2}, \cdots$, with $a_{i}$ interior to $S_{i}$, such that, no matter how fast the sequence of numbers $m_{1}, m_{2}, \cdots$ increases there will be an integral function $f(z)$ for which

$$
|f(t)| \geqq m_{i} \text { in } S_{i} \text { ? }
$$

In Part II we have shown that this is actually the case; but our construction will ordinarily give a function $f(z)$ with may zeros. This makes our desired

Presented to the Society, December 29, 1936, under the title On the possible behavior of an analytic function at a set of isolated points; received by the editors August 25, 1939, and, in revised form, June 3, 1940.

(1) Most of this paper was written while the writer was a member of the Institute for Advanced Study. tions.

The writer is greatly indebted to Professors Bochner and Bohnenblust for valuable sugges-

(2) See $\$ 11$ of this paper.

(3) American Journal of Mathematics, vol. 14 (1892), p. 214.

${ }^{(4)}$ Lȩ̧ons sur les Séries à Termes Positifs, Paris, 1902, p. 27. 
level curve $C$ have not only the required branches, but many extraneous ones in addition. Whether or not there exists a nonvanishing function $f(z)$ with the stated properties, so that the corresponding level curve $C$ has exactly the required branches and no more, is a question that we have been unable to settle.

On investigating what sort of regions $S_{1}, S_{2}, \cdots$ may be used to obtain the required properties, certain questions concerning the topology on a sphere arose; these are considered in Part I. In so doing, it was found expedient to make certain restatements of Hilbert's theorem on the approximation of Jordan curves by lemniscates.

\section{PART I}

1. Preliminary definitions. By a ring on the extended complex plane or the Riemann sphere, we mean an open or closed region bounded by two Jordan curves that have no point in common. The point at infinity may be interior to, exterior to, or on the boundary of the ring.

The only sets or sequences of rings considered in this paper are those in which the rings are mutually non-intersecting; that is, no two of the rings have closures with points in common. For this reason we shall usually not bother to state explicitly the non-intersecting character of the rings, but this condition is always to be understood.

Topologically, there is no distinction between a given ring on a sphere and any other ring on a sphere. Likewise every pair of rings is equivalent to every other pair; the complementary regions into which the rings divide the plane necessarily consist of two simply connected regions $A$ and $B$ and a third ring $R$ which separates $A$ from $B$. For three or more rings, on the other hand, we may distinguish relative positions according to the arrangement and connectivity of the complementary regions. If there are $n$ given rings, the connectivity $k$ of the complementary region of maximum connectivity may vary from 2 to $n$. We distinguish the two extreme cases by names: if $k$ is 2 we call the rings nested, if $k=n$ the rings are mutually exterior. (This must not be confused with mutually exterior in the point-set-theoretic sense, which means non-intersecting in our terminology.)

The multiply connected region which is complementary to a set of $n$ mutually exterior rings will be called the $R$-exterior of the rings; the other complementary regions, all simply connected and $n$ in number, the $R$-interiors.

The same definitions of nested, mutually exterior, $R$-exterior, and $R$-interior will evidently apply as well to finite sets of non-intersecting Jordan curves on the sphere.

2. Generalizations of Hilbert's theorem. According to Walsh and Russell's $\left(^{5}\right)$ generalization of a theorem due to Hilbert, a finite number of mu-

(5) These Transactions, vol. 36 (1934), pp. 13-28. 
tually exterior Jordan curves whose $R$-exterior contains the point at infinity can be uniformly approximated by means of lemniscates.

As thus stated, Hilbert's theorem imposes a special rôle on the point at infinity. To avoid this, we generalize the notion of lemniscate as follows: A lemniscate with poles at $b_{1}, \cdots, b_{n}$ and zeros at $a_{1}, \cdots, a_{m}$ is a level curve of a rational function whose poles are at $b_{1}, \cdots, b_{n}$ and whose zeros are at $a_{1}, \cdots, a_{m}$.

By imposing the transformation $z=1 /\left(z^{\prime}-b\right)$ in Hilbert's theorem, we get the following more symmetrical statement: Any set of mutually exterior Jordan curves can be uniformly approximated by a lemniscate with pole at $b$ and zeros at $a_{1}, \cdots, a_{m}$, where $b$ is a preassigned point in the $R$-exterior and the $a$ 's are somewhere in the $R$-interiors of the given curves.

This statement suggests a dual theorem in which the rôles of pole and zeros are interchanged. To get such a dual theorem we may first consider the reciprocal of the rational function yielding the lemniscate in the last statement. It follows that the given Jordan curves can be approximated by a lemniscate with zero at $b$ and poles at $a_{1}, \cdots, a_{m}$. Now, applying Runge's method of moving of poles, we may approximate our rational function uniformly in the $R$-exterior of the curves by another rational function with poles at given points $b_{1}, \cdots, b_{n}$, one in each of the $R$-interiors of the curves. The degree of approximation being arbitrary, one may also choose this new function so that its zeros lie as close as one pleases to $b$. Repeating this process, we obtain the following four theorems, of which Theorem $\mathrm{I}$ is a sharpening of Hilbert's theorem.

TheOREM I [TheOREM I']. Let $R_{1}, \cdots, R_{n}$ be given mutually exterior rings on the extended complex plane, and let $b$ be a given point in the $R$-exterior of the rings and $a_{1}, \cdots, a_{n}$ given points such that $a_{i}$ is in the $R$-interior of $R_{i}$.Then there is a lemniscate, whose only pole [zero] is at $b$, which consists of $n$ Jordan curves, $C_{1}, \cdots, C_{n}$, with $C_{i}$ interior to $R_{i}$ (in the point-set-theoretic sense) and with $C_{i}$ separating the $R$-interior of $R_{i}$ from its $R$-exterior. Furthermore, the zeros [poles] of the lemniscate may be taken at a set of points $c_{i, j}, i=1, \cdots, n$, $j=1, \cdots, l_{i}$, such that the distance from any $c_{i, j}$ to $a_{i}$ is less than $\epsilon$, where $\epsilon$ is an arbitrarily small positive number.

TheOREM II [TheOREM II']. Under the same hypotheses as in Theorem I, there is a lemniscate with poles [zeros] at $a_{1}, \cdots, a_{n}$ which consists of $n$ Jordan curves $C_{1}, \cdots, C_{n}$ with the same properties as in Theorem I. Furthermore, the zeros [poles] of the lemniscate can be taken at points $c_{1}, \cdots, c_{l}$, where each $c_{i}$ is at distance less than $\epsilon$ from $b$.

3. Further definitions. A point $b$ will be called a sequential limit point of a sequence of rings $R_{1}, R_{2}, \cdots$ if there is a sequence of points $\beta_{1}, \beta_{2}, \cdots$, with $\beta_{i}$ on $R_{i}$, which has $b$ as a limit point. 
More generally, if $\left\{S_{\nu}\right\}$ is an infinite collection of mutually disjunct sets, either denumerable or nondenumerable in number, then a point $b$ will be called a sequential limit point of the collection if there is a sequence of points $\beta_{1}, \beta_{2}, \cdots$ such that (i) every $\beta_{i}$ is in some $S_{\nu}$, (ii) no two of the $\beta$ 's are in the same $S_{\nu}$, (iii) the sequence of $\beta$ 's has $b$ as a limit point.

It can be shown that the rings $R_{1}, R_{2}, \cdots, R_{n}$ are nested if and only if they can be ordered in such a way that $R_{i}$ separates $R_{j}$ from $R_{k}$ whenever $i$ is between $j$ and $k$.

An infinite sequence of rings will be called nested if it can be arranged in an order $R_{1}, R_{2}, \cdots$ such that $R_{i}$ separates $R_{j}$ from $R_{k}$ whenever $i$ is between $j$ and $k$. An equivalent definition is that, when properly ordered, $R_{j}$ separates $R_{k}$ from the set of sequential limit points whenever $j$ is greater than $k$.

We shall denote the boundary of a set $A$ by $F(A)$ and its complement by $C(A)$.

An interior sequential limit point of a sequence of rings is a sequential limit point of a nested subsequence of the rings.

Every closed set $B$, not the null set or the whole plane, divides the plane into a finite number or denumerable infinity of domains $\sigma_{1}, \sigma_{2}, \cdots$ namely, the components of $C(B)$. A closed set $B$ will be said to be encased in a sequence of rings in $C(B)$ if, for each $i$, those rings which are in $\sigma_{i}$ form a subsequence with the following two properties:

(a) The set of sequential limit points, the interior sequential limit points, and $F\left(\sigma_{i}\right)$ are three identical sets.

(b) If $F\left(\sigma_{i}\right)$ has more than one component, then for every ring there are points of $F\left(\sigma_{i}\right)$ in both of the two regions complimentary to the ring. If $F\left(\sigma_{i}\right)$ has only one component, then the rings are nested.

\section{A theorem of plane topology.}

TheOREM III. Let $B$ be any closed set, not the null set or the whole plane, and let $S$ be a set in $C(B)$ whose components $\left\{S_{\nu}\right\}$ are closed and have sequential limit points only in $B$. Then there exists a sequence of rings in $C(B+S)$ which encases $B$. The boundary curves of these rings may be taken to be lemniscates.

Proof. We assume a metric for the entire extended plane, as, for instance, distances on the Riemann sphere.

For the proof it will be sufficient to show that if $\sigma_{i}$ is any component of $C(B)$, then there exists in $\sigma_{i}-S \sigma_{i}$ a sequence of rings which satisfies the above condition (b) and whose sequential limit points and interior sequential limit points are both identical with $F\left(\sigma_{i}\right)$.

If $z$ is a point in a non-null set $A$ with a non-null boundary, we shall call its distance from $F(A)$ the depth of $z$ in $A$. In any non-null set $A$ there will be one or more points which have maximum depth $\rho_{0} \geqq 0$ in $A$. In particular, if $A$ is open, then there will be a point having maximum depth $\rho_{0}>0$. The number $\rho_{0}$ will be called the maximum depth of the set $A$. Since our metric is 
bounded, every set has finite maximum depth. If $\rho_{0}$ is the maximum depth of $A$, then for every number $\rho, 0 \leqq \rho \leqq \rho_{0}$ there are points in $A$ with depth $\rho$. If $B$ is a closed set in $A$, we shall call the greatest depth of any point of $B$ in $A$ the maximum depth of $B$ in $A$ and the smallest depth of any point of $B$ in $A$ the minimum depth of $B$ in $A$.

The components of $S$ which have points in common with $\sigma_{i}$ are entirely in $\sigma_{i}$. Let $K(\rho)$ be the sum of the components of $S$ whose minimum depth in $\sigma_{i}$ is larger than $\rho$, where $\rho$ is any positive number less than the maximum depth $\rho_{0}$ of $\sigma_{i}$. There will be at most a finite number of components of $S$ in $K(\rho)$ : otherwise there would be a sequential limit point of the components at a positive depth in $\sigma_{i}$, which is contrary to hypothesis. Let $k(\rho)$ be the sum of the components of $S$ whose minimum depth in $\sigma_{i}$ is not greater than $\rho$. Let $A(\rho)$ be the set of all points whose depth in $\sigma_{i}$ is greater than $\rho$. The boundary $F(A(\rho))$ is non-vacuous. Let $a(\rho)$ be the set of all points whose depth in $\sigma_{i}$ is not greater than $\rho$.

We will now show that if $0<\rho_{1}<\rho_{0}$ then there is a number $\rho_{2}, 0<\rho_{2}<\rho_{1}$, such that $a\left(\rho_{2}\right)+k\left(\rho_{2}\right)$ is at a positive distance, say $\delta$, from $A\left(\rho_{1}\right)+K\left(\rho_{2}\right)$. Obviously $a\left(\rho_{2}\right)$ is at a positive distance from $A\left(\rho_{1}\right)$ and from $K\left(\rho_{2}\right)$, and $k\left(\rho_{2}\right)$ from $K\left(\rho_{2}\right)$. Suppose no number $\rho_{2}$ exists such that $k\left(\rho_{2}\right)$ is at a positive distance from $A\left(\rho_{1}\right)$. No fixed component of $S$ can be in $k\left(\rho_{2}\right)$ for every $\rho_{2}$; hence there must exist a sequence of components $S_{1}, S_{2}, \cdots$ such that the distance from $S_{j}$ to $A\left(\rho_{1}\right)$ approaches zero with $j$. But this would imply a sequential limit point of the $S_{j}$ at positive depth in $\sigma_{i}$, which is contrary to hypothesis.

Cover the closure of $a\left(\rho_{2}\right)+k\left(\rho_{2}\right)$ by a finite number of circular closed regions with centers on this set and radius $\eta<\delta$. The number $\eta$ can be chosen so that no two of the circular boundaries of these regions will be tangent to each other, and no three intersect at a single point. Let $L(\eta)$ be the sum of these circular regions.

If we start at any point on the boundary of $L(\eta)$ and proceed along the boundary, we will arrive back at the starting point after traversing a finite number of arcs of circles and an equal number of points where two circles intersect. The boundary of $L(\eta)$ consists of a finite number of Jordan curves. Furthermore, it is possible to increase the radius of each circle in $L(\eta)$ slightly without changing the connections between the circular arcs forming the boundary of $L(\eta)$. Otherwise, the connections would change in a discontinuous manner; but this can happen only when two arcs are tangent or when more than two arcs intersect at a point. Hence there is an $\eta^{\prime}$ between $\eta$ and $\delta$ such that $\left[L\left(\eta^{\prime}\right)-L(\eta)\right] \sigma_{i}$ consists of a finite number of rings $R_{1}, R_{2}, \cdots, R_{k}$. By their construction, these rings have a minimum depth in $\sigma_{i}$ greater than $\rho_{2}$, a maximum depth less than $\rho_{1}$, and are at a positive distance from $S$.

A ring $R$ will be said to bound a set $A, A R \neq 0$, if one of the Jordan curves forming the boundary of $R$ is contained in $F(A)$. A collection of rings $R_{1}, R_{2}, \cdots, R_{p}$ will be said to completely bound $A$ if each of the rings bounds 
$A$ and if $F(A)$ is contained in the closure of the rings. A set of rings which completely bounds a connected set will be mutually exterior and will separate $A$ from $C\left(A+\sum R_{j}\right)$.

The rings $R_{1}, R_{2}, \cdots, R_{k}$ will completely bound $\sigma_{i}-L\left(\eta^{\prime}\right) \sigma_{i}$. Let $M$ be a component of $\sigma_{i}-L\left(\eta^{\prime}\right) \sigma_{i}$ which contains a point $x_{0}$ of maximum depth $\rho_{0}$ in $\sigma_{i}$. There will be a subset of the rings, $R_{1}, R_{2}, \cdots, R_{p}$, which completely bound $M$. These rings will be mutually exterior and will separate $M$ from $F\left(\sigma_{i}\right)$.

If $x$ is any point in $\sigma_{i}$, it can be joined to $x_{0}$ by a Jordan arc in $\sigma_{i}$. This arc will have a positive distance $\lambda$ from $F\left(\sigma_{i}\right)$, that is, it will have positive minimum depth $\lambda$ in $\sigma_{i}$. Hence there exists a $\rho_{1}$ so small that the corresponding set $M$ will contain $x$, and the corresponding rings $R_{1}, \cdots, R_{p}$ will separate $x$ from $F\left(\sigma_{i}\right)$. If $F\left(\sigma_{i}\right)$ is connected, then $M$ will be simply connected for every $\rho_{1}$. On the other hand, if $F\left(\sigma_{i}\right)$ is not connected, then $M$ will be multiply connected for every sufficiently small $\rho_{1}$.

Let $\epsilon_{1}$ be less than $\rho_{0}$ and also so small that if $F\left(\sigma_{i}\right)$ is not connected then the set $M$ corresponding to any $\rho_{1} \leqq \epsilon_{1}$ will be multiply connected. Starting with $\epsilon_{1}$ we form any sequence $\epsilon_{1}, \epsilon_{2}, \ldots$ of positive numbers with zero as a limit and such that if $\epsilon_{j}$ is taken to be $\rho_{1}$ in the above, then $\rho_{2}$ may be taken equal to $\epsilon_{j+1}$. That is, $a\left(\epsilon_{j+1}\right)+k\left(\epsilon_{j+1}\right)$ is at a positive distance from $A\left(\epsilon_{j}\right)$ $+K\left(\epsilon_{j+1}\right)$. Corresponding to each pair of values $\epsilon_{j}, \epsilon_{j+1}$ there will be a set of rings $R_{1}^{(j)}, \cdots, R_{p}^{(j)}$ constructed as above on taking $\rho_{1}=\epsilon_{j}$ and $\rho_{2}=\epsilon_{j+1}$.

It will now be shown that the totality of these rings is a sequence which encases $F\left(\sigma_{i}\right)$.

(i) That condition (b) will be satisfied is a consequence of the fact that the rings $R_{1}^{(j)}, \cdots, R_{p}^{(j)}$ completely bound a set $M_{j}$ which is simply connected if $F\left(\sigma_{i}\right)$ has only one component and multiply connected otherwise.

(ii) Every sequential limit point of the rings is on $F\left(\sigma_{i}\right)$ because $R_{k}^{(j)}$ has maximum depth less than $\epsilon_{j}$ and $\epsilon_{j} \rightarrow 0$.

(iii) Every point of $F\left(\sigma_{i}\right)$ is a sequential limit point of the rings. If not, then there exists a point $x$ in $F\left(\sigma_{i}\right)$ and a circular neighborhood $N_{x}$ such that $N_{x}$ is free of points of the rings. But $N_{x}$ contains at least one point $y$ in $\sigma_{i}$. For $j$ sufficiently great, $R_{k}^{(j)}$ separates $y$ from $x$. This can happen only if $R_{k}^{(j)}$ has points in $N_{x}$, which is a contradiction.

(iv) It remains to show that every point of $F\left(\sigma_{i}\right)$ is an interior sequential limit point of the rings; that is, for every $x$ in $F\left(\sigma_{i}\right)$ there is a nested subsequence of the rings which has $x$ as a sequential limit point. Since the rings $R_{1}^{(j)}, \cdots, R_{p}^{(j)}$ separate $x_{0}$ from $F\left(\sigma_{i}\right)$, there will be a particular ring, say $R_{\mu_{j}}^{(j)}$, which separates $x_{0}$ from $x$. The sequence of rings $\left\{R_{\mu_{j}}^{(j)}\right\}$ will have $x$ as a sequential limit point and will be nested.

That the boundary curves of the rings can be taken to be lemniscates follows immediately from Theorem II and the fact that the rings $R_{1}^{(j)}, \cdots, R_{p}^{(j)}$ are mutually exterior. In fact, the poles of the lemniscate may be taken on $F\left(\sigma_{i}\right)$, since if $p>1$ each ring contains a point of $F\left(\sigma_{i}\right)$ in its $R$-interior. 


\section{PART II}

\section{Construction of functions with very rapid growth.}

TheOREM IV. Let $G(z) \geqq 0$ be a given function defined for every point $z$ in the extended complex plane. Let $B$ be the set consisting of all points in every neighborhood of which $G(z)$ is unbounded. We suppose that $B$ is neither the null set nor the whole extended plane. Let $R_{1}, R_{2}, \cdots$ be any sequence of mutually nonintersecting rings which encases $B$. Then there exists a function $f(z)$ which is analytic except possibly at points of $B$ and which satisfies the inequality

$$
|f(z)| \geqq G(z)
$$

for every $z$ not in one of the rings.

Corollary. Let $G(z)$ and $B$ be given as in Theorem IV. Let $S$ be any set in $C(B)$ whose components are closed and whose sequential limit points are all on $B$. Then there is a function $f(z)$ which is analytic except on $B$ and which satisfies the inequality (1) at all points of $S$. IV.

By Theorem III, this corollary is an immediate consequence of Theorem

In this section and throughout the paper, all functions are assumed to be single valued.

In case $G(z)$ is bounded in the whole plane ( $B$ is the null set), the problem is trivial; since one can then satisfy (1) everywhere by taking $f(z)$ to be a sufficiently large constant.

Proof of Theorem IV. We confine our attention to one of the domains $\sigma_{k}$ into which $B$ divides the plane, and to those rings which lie in $\sigma_{k}$. Let $P$ be any point in $\sigma_{k}$ and not in any of the rings. Then we distinguish the two parts of the plane exterior to any ring $R_{j}$ by the inside and outside of $R_{j}$ according to whether it does or does not contain $P$, respectively. Having chosen $P$, every ring has an inside and outside, but different choices of $P$ may interchange the inside and outside of a given ring. We suppose that $P$ is now chosen once for all, subject only to the condition that if the complement $S$ of the rings in $\sigma_{k}$ has a simply connected component $S_{0}$ (which can happen only if $F\left(\sigma_{k}\right)$ is connected), then $P$ is in $S_{0}$.

If a nested subsequence of the rings in $\sigma_{k}$ has a point $x$ on $F\left(\sigma_{k}\right)$ as a sequential limit point, then any point $y$ in $\sigma_{k}$ will be separated from $x$ by all but a finite number of this subsequence of rings. For, let $y$ be a point in $\sigma_{k}$ which is not separated from $x$ by an infinity of rings. Let $z$ be a point in $\sigma_{k}$ which is separated from $x$ by the first of the rings. Join $z$ to $y$ by a Jordan arc in $\sigma_{k}$. Every ring separates $z$ from $x$. There must be an infinite number of rings which separate $y$ from $z$. These rings will all intersect the Jordan arc $y z$; therefore, there will be a sequential limit point of the rings on the arc, and hence in $\sigma_{k}$; which is a contradiction. 
Let $S$ be the set consisting of all points in $\sigma_{k}$ which are not in any ring. Each component $S_{j}$ of $S$ is completely bounded by a finite number of the rings. For, if an infinite number of rings bound $S_{j}$, then there will be a sequential limit point $x$ of the rings in the closure of $S_{j}$. This point $x$ will also be on $F\left(\sigma_{k}\right)$ and hence will be a sequential limit point of a nested subsequence of the rings. Any point $y$ of $S_{j}$ is in $\sigma_{k}$. We have just seen that $y$ is separated from $x$ by all but a finite number of rings. But this is a contradiction since $S_{j}+x$ is a connected set, in the complement of the rings, which contains $x$ and $y$.

Denote the component of $S$ which contains $P$ by $S_{0}$. There will be only a finite number of rings which separate any component from $P$, and only a finite number of components, $S_{i 1}, \cdots, S_{i l_{i}}$, each of which is separated from $P$ by exactly $i$ rings. We now rename the rings by using a double subscript $R_{i j}$, $j=1,2, \cdots, l_{i}$, so that $S_{i j}$ is outside of and bounded by $R_{i j}$. Let $I_{i j}$ be the inside of $R_{i j}$. Put

$$
I^{(i)}=I_{i 1} \cdot I_{i 2} \cdots I_{i l_{i}}, \quad S^{(i)}=S_{i 1}+S_{i 2}+\cdots+S_{i l_{i}} .
$$

The rings $R_{i 1}, \cdots, R_{i l_{i}}$ are mutually exterior, since they completely bound the connected set $I^{(i)}$; and there will be points of $F\left(\sigma_{k}\right)$ outside each of these rings.

Let $M_{0}$ be the upper bound of $G(z)$ in $S_{0}$ and $M_{i}$ the upper bound in $S^{(i)}$.

Let $\epsilon_{1}, \epsilon_{2}, \cdots$ be any set of positive numbers such that $\sum \epsilon_{i}$ converges to a given sum $\epsilon$.

Let $N_{1}$ be the positive constant $M_{0}+\epsilon$. By Theorem II there is a rational function $F_{1}(z)$, analytic except at points of $B$, such that the lemniscate $\left|F_{1}(z)\right|=1$ consists of $l_{1}$ contours $C_{11}, \cdots, C_{i l_{1}}$, where $C_{1 j}$ is interior to $R_{1 j}$ and separates $S_{1 j}$ from $P, j=1,2, \cdots, l_{i}$. Then there will exist a positive integer $p_{1}$ so large that

$$
\begin{aligned}
& \left|F_{1}(z)\right|^{p_{1}}<\epsilon_{1} \quad \text { in } S_{0}=I^{(1)}, \\
& >M_{1}+N_{1}+\epsilon \text { in } S^{(1)} \text {. }
\end{aligned}
$$

Let $N_{2}$ be the upper bound of $\left|N_{1}+\left[F_{1}(z)\right] p_{1}\right|$ in $S^{(2)}$.

Similarly, there is a rational function $F_{2}(z)$, whose only poles are on $B$, such that the lemniscate $\left|F_{2}(z)\right|=1$ consists of $l_{2}$ contours $C_{21}, \cdots, C_{2 l_{2}}$, where $C_{2 j}$ is interior to $R_{2 j}$ and separates $S_{2 j}$ from $P$. Then there will be a positive integer $p_{2}$ so large that

$$
\begin{aligned}
& \left|F_{2}(z)\right|^{p_{2}}<\epsilon_{2} \quad \text { in } I^{(2)} \text {, } \\
& >M_{2}+N_{2}+\epsilon \text { in } S^{(2)} \text {. }
\end{aligned}
$$

Continuing this process, we suppose that $F_{3}(z), \cdots, F_{i-1}(z)$ have been constructed. Let

$$
V_{i+1} \geqq\left|N_{1}+\left[F_{1}(z)\right]^{p_{1}}+\left[F_{2}(z)\right]^{p_{2}}+\cdots+\left[F_{i-1}(z)\right]^{p_{i-1}}\right|
$$


in $S^{(i)}$. There is a rational function $F_{i}(z)$, whose only poles are on $B$, such that the lemniscate $\left|F_{i}(z)\right|=1$ will consist of $l_{i}$ contours $C_{i 1}, C_{i 2}, \cdots, C_{i l_{i}}$, where $C_{i j}$ is interior to $R_{i j}$ and separates $S_{i j}$ from $P$. Then there will be a positive integer $p_{i}$ so large that

$$
\begin{aligned}
\left|F_{i}(z)\right|^{p_{i}} & <\epsilon_{i} & \text { in } I^{(i)} \\
& >M_{i}+N_{i}+\epsilon & \text { in } S^{(i)} .
\end{aligned}
$$

Now consider the function

$$
f(z)=N_{1}+\sum_{i=1}^{\infty}\left[F_{i}(z)\right]^{p_{i}}
$$

Let $\Sigma$ be any closed region exterior to $B$, and let $z$ be a point of $\Sigma$. From the manner in which the terms of (2) were constructed, it is clear that one can find an integer $q$ so large that

$$
\left|F_{i}(z)\right|^{p_{i}}<\epsilon_{i} \quad \text { for } z \text { in } \Sigma \text { and } i>q .
$$

Since $\sum \epsilon_{i}$ converges, this shows that the series (2) converges uniformly in $\Sigma$. Hence $f(z)$ is analytic everywhere except for the points of $B$.

Moreover, for $z$ in one of the regions $S_{q 1}, S_{q 2}, \cdots, S_{q l}$, one can write

$$
\begin{aligned}
|f(z)| & \geqq\left|F_{q}(z)\right|^{p_{q}}-\left|N_{1}+\sum_{i=1}^{q-1}\left[F_{i}(z)\right]^{p_{i}}\right|-\sum_{i=q+1}^{\infty}\left|F_{i}(z)\right|^{p_{i}} \\
& \geqq\left(M_{q}+N_{q}+\epsilon\right)-N_{q}-\sum_{i=q+1}^{\infty} \epsilon_{i} \\
& \geqq M_{q} \geqq G(z),
\end{aligned}
$$

and the theorem follows.

6. Best possible character of Theorem IV. In Theorem IV the rings $R_{1}, R_{2}, \cdots$ present a sort of barrier between the set $B$, where $G(z)$ is unbounded, and the set $S$, where (1) holds. It is true that points of $B$ may be limit points of $S$, but no point of $B$ can be a limit point of points in any'single region in $S$. Thus, collectively the regions of $S$ are close to $B$, but individually they are not.

The question arises as to whether some such barrier is necessary. We answer this question in the affirmative in the following theorem, which states that no theorem like Theorem IV can be true in case a region in $S$ has a limit point on $B$.

THEOREM V. Let $B$ be any closed set, not the null set or the whole plane, and let $S$ be an open region which has at least one point of $B$ on its boundary, but has no points in common with $B$. Then there exists a function $G(z)$ such that $B$ consists of precisely those points in every neighborhood of which $G(z)$ is unbounded, 
and such that no function $f(z)$ exists which is meromorphic in $S$ and satisfies (1) in $S$.

Proof. Let $C_{1}, C_{2}, \cdots$ be a nested sequence of analytic Jordan curves in $S$, having a point $b$ of $B$ as an interior sequential limit point, and such that the open regions $\Sigma_{1}, \Sigma_{2}, \cdots$ in $S$ bounded by these contours all have an interior point in common, say $s_{1}$. Then there exists a sequence of mapping functions which will map $\Sigma_{1}, \Sigma_{2}, \cdots$ conformally onto the interior of the unit circle in such a way that the origin is the image of $s_{1}$. The sequence of one-to-one transformations thus defined will transform any function $f(z)$, meromorphic in $S$, into a sequence of functions $\left\{f_{i}(z)\right\}$ which are meromorphic in and on the unit circle.

Let $\theta_{1}, \theta_{2}, \cdots$ be a sequence of arcs on the unit circle such that the corresponding sequence of $\operatorname{arcs} \theta_{1}^{\prime}, \theta_{2}^{\prime}, \cdots$ on the curves $C_{1}, C_{2}, \cdots$ will have $b$ as its only sequential limit point. For brevity, we also denote by $\theta_{1}, \theta_{2}, \cdots$ the lengths of the $\operatorname{arcs} \theta_{1}, \theta_{2}, \cdots$. Let $M_{1}, M_{2}, \cdots$ be any sequence of positive constants such that $\theta_{i} \log M_{i} \rightarrow \infty$ as $i \rightarrow \infty$.

Let $G(z)$ be defined as equal to unity in $S$ except on the $\operatorname{arcs} \theta_{1}^{\prime}, \theta_{2}^{\prime}, \ldots$; where we take $G(z)=M_{1}, M_{2}, \cdots$, respectively. Outside $S, G(z)$ is given any values such that $G(z) \rightarrow \infty$ if and only if $z$ tends to a point of $B$. The points $B$ will be precisely those in every neighborhood of which this function $G(z)$ is unbounded.

We now show that no function $f(z)$ can exist which is meromorphic in $S$ and satisfies (1) in $S$. Suppose, on the contrary, that such a function did exist. Then the transformed functions $f_{i}(z)$ would be meromorphic in and on the unit circle and $\left|f_{i}(z)\right| \geqq G_{i}(z)$ for $|z| \leqq 1$, where the $G_{i}(z)$ are the transforms of $G(z)$. Hence, by Jensen's theorem, since $\left|f_{i}(z)\right| \geqq 1$ for $|z| \leqq 1$,

$$
\log \left|f_{i}(0)\right|=\frac{1}{2 \pi} \int_{0}^{2 \pi} \log \left|f_{i}\left(e^{i \theta}\right)\right| d \theta+\sum_{p} \log \frac{1}{\left|b_{i p}\right|},
$$

where the $b_{i p}$ are the poles of $f_{i}(z)$ in the unit circle. The last term is not negative, and the integral is larger than $\theta_{i} \log M_{i}$; so that

$$
\log \left|f_{i}(0)\right|=\log \left|f\left(s_{1}\right)\right| \geqq \frac{1}{2 \pi} \theta_{i} \log M_{i} .
$$

The right-hand member of this equation tends to infinity as $i$ becomes infinite, while the left-hand member is fixed, independent of $i$. We thus have a contradiction, and the theorem is proved.

7. Generalizations of Theorems IV and V. The last two theorems may be generalized by replacing the inequality (1) by other relationships. We may, for instance, let $g(z)$ be a given analytic function and require that

$$
f(z)=g(z)+h(z), \quad|h(z)| \leqq G(z) .
$$


This problem is one of approximation to analytic functions by means of analytic functions, the degree of approximation to be predetermined by the given function $G(z)$. We thus have

THEOREM IV'. Let $G(z)$ be a given function which is defined and positive in the entire complex plane. Let $B$ be the set of points in every neighborhood of which $G(z)$ has zero as a greatest lower bound. We assume that $B$ is neither the null set nor the whole extended plane. Let $R_{1}, R_{2}, \cdots$ be any sequence of rings which encases $B$, and $g(z)$ any function which is analytic for all $z$ not in $B$. Then there is a function $f(z) \not \equiv g(z)$ which is meromorphic except at points of $B$ and which satisfies $\left(1^{\prime}\right)$ for every $z$ not in one of the rings.

Theorem $V^{\prime}$. Let $B$ be a given closed set, not the null set or the whole extended plane, and let $S$ be an open region which has at least one point of $B$ on its boundary, but has no points in common with $B$. Let $g(z)$ be a given function which is analytic for every $z$ not in $B$. Then there exists a function $G(z)>0$ such that $B$ consists of precisely those points in every neighborhood of which $G(z)$ has zero as a greatest lower bound, and such that no function $f(z)$ exists which is meromorphic in $S$ and satisfies $\left(1^{\prime}\right)$ in $S$.

These two theorems are obtained immediately when one applies Theorems IV and V, respectively, to the reciprocals of $h(z)$ and $G(z)$.

8. An improvement of Theorem IV in a special case. In the next theorem we present an example of a situation intermediate between Theorems IV and $\mathrm{V}$. The theorem involves a certain kind of set $S$ which resembles a cartwheel and which is described as follows: (a) The set $S$ includes all points in an infinite sequence of concentric, circular rings with centers at the origin, which has infinity as its only sequential limit point. (b) Let $\alpha_{1}, \alpha_{2}, \ldots$ be a sequence of rays from the origin whose angles with the real axis are rational multiples of $2 \pi$, and let $r_{1}, r_{2}, \cdots$ be positive numbers with $r_{i} \rightarrow \infty$; then $S$ includes all those points on $\alpha_{i}$ where $|z| \geqq r_{i}, i=1,2, \cdots$.

TheOREM VI. Let $S$ be a given set of points of the sort just described. Let $G(z)$ be any given positive valued function which is bounded in every bounded region of the plane. Then there exists an integral function $f(z)$ such that (1) is satisfied everywhere on $S$.

Proof. Denote the rings of $S$ by $R_{1}, R_{2}, \cdots$. Without loss of generality we may assume that the $r$ 's are non-decreasing. Denote by $l_{i}$ the last value of $j$ such that $z=r_{j}$ is inside $R_{i}$. (This use of "inside" agrees with our previous use of the term if we take $P$ to be the origin.) Let $S_{0}$ consist of those points of the rays $\alpha_{1}, \alpha_{2}, \cdots$ which are inside $R_{1}$. Let $S_{1}$ consist of the points of $R_{1}$ together with the points of the rays which are outside $R_{1}$ and inside $R_{2}$; and $S_{k}$ the points of $R_{k}$ together with those of the rays which are between $R_{k}$ and $R_{k+1}$. Let $\epsilon_{1}, \epsilon_{2}, \cdots$ be a sequence of positive numbers such that $\sum \epsilon_{i}$ con- 
verges to a given value $\epsilon$. Let $M_{i}$ be the upper bound of $G(z)$ in $S_{i}$. Let $a_{i}$ be the radius of the smaller of the two circles bounding $R_{i}$.

Put $N_{0}=M_{0}+\epsilon, N_{1}=M_{1}+N_{0}+\epsilon$. For brevity, we also denote by $\alpha_{1}, \alpha_{2}, \ldots$ the fractional multiplies of $2 \pi$ that the rays $\alpha_{1}, \alpha_{2}, \cdots$ make with the axis of reals. We choose $p_{1}$ to be a positive integer such that $p_{1} \alpha_{j}$ is an integer for $j=1,2, \cdots, l_{2}$. Let

$$
N_{2}=\underset{Z \text { in } S_{2}}{\operatorname{lou}}\left\{M_{2}+\epsilon+\left|N_{0}+N_{1}\left(\frac{z}{a_{1}}\right)^{p_{1}}\right|\right\} \text {. }
$$

We choose $p_{2}$ to be a positive integer such that (a) $p_{2} \alpha_{j}$ is an integer for $j=1,2, \cdots, l_{3}$, and (b) $\left|N_{2}\left(z / a_{2}\right)^{p_{2}}\right|$ is less than $\epsilon_{2}$ inside and on $R_{1}$.

Continuing this process, at the typical stage we let

$$
N_{i}=\underset{Z \text { in } S_{i}}{\text { l.u.b. }}\left\{M_{i}+\epsilon+\left|N_{0}+N_{1}\left(\frac{z}{a_{i}}\right)^{p_{1}}+\cdots+N_{i-1}\left(\frac{z}{a_{i-1}}\right)^{p_{i-1}}\right|\right\} \text {. }
$$

Then we take $p_{i}$ to be a positive integer so large that (a) $p_{i} \alpha_{j}$ is an integer for $j=1,2, \cdots, l_{i+1}$, and (b) $\left|N_{i}\left(z / a_{i}\right)^{p_{i}}\right|$ is less than $\epsilon_{i}$ inside and on $R_{i-1}$.

Now consider the function

$$
f(z)=N_{0}+\sum_{i=1}^{\infty} N_{i}\left(\frac{z}{a_{i}}\right)^{p_{i}} .
$$

Let $\Sigma$ be any finite closed region, and $z$ a point of $\Sigma$. Then there is a $q$ so large that

$$
\left|N_{i}\left(\frac{z}{a_{i}}\right)^{p_{i}}\right|<\epsilon_{i} \quad \text { for } z \text { in } \Sigma \text { and } i>q .
$$

Since $\Sigma \epsilon_{i}$ converges, this means that (3) converges uniformly in $\Sigma$, and $f(z)$ is an integral function.

Moreover, if $z$ is in one of the rings $R_{q}$, we can write

$$
\begin{aligned}
|f(z)| & \geqq\left|N_{q}\left(\frac{z}{a_{q}}\right)^{p_{q}}\right|-\left|N_{0}+\sum_{i=1}^{q-1} N_{i}\left(\frac{z}{a_{i}}\right)^{p_{i}}\right|-\left|\sum_{i=q+1}^{\infty} N_{i}\left(\frac{z}{a_{i}}\right)^{p_{i}}\right| \\
& \geqq M_{q} \geqq G(z) .
\end{aligned}
$$

If $z$ is on one of the rays and in $S_{q}$, then

$$
|f(z)| \geqq \sum_{i=q}^{\infty} N_{i}\left(\frac{z}{a_{i}}\right)^{p_{i}}-\left|N_{0}+\sum_{i=1}^{q-1} N_{i}\left(\frac{z}{a_{i}}\right)^{p_{i}}\right| \geqq M_{q} \geqq G(z) .
$$

This concludes the proof of the theorem.

9. An unsolved problem. As mentioned in the Introduction, a problem, intermediate between Theorems IV and V, which the writer has been unable to answer, but whose solution could be used to advantage in the subsequent 
part of this paper, is the following: Let $S_{1}, S_{2}, \cdots$ be a given infinite sequence of simply connected regions whose closures are non-intersecting and whose only sequential limit point is the point at infinity. Let $M_{1}, M_{2}, \cdots$ be a given sequence of positive numbers. Then, does there exist a nonvanishing integral function, or even a nonvanishing meromorphic function, $f(z)$, such that

$$
|f(z)| \geqq M_{i}
$$

for $z$ in $S_{i}$ ? The available evidence leads to the conjecture that for every sequence of regions there will correspond $M$ 's for which no such integral function exists.

10. A theorem on level curves. The zeros of an analytic function are isolated. Conversely, according to Mittag-Leffler's theorem, given any isolated - set $I$, there is a function which has a zero at each point of $I$ and none elsewhere and which is analytic except at limit points of $I$.

The singularities of an analytic function form a closed set. For any given closed set $B$ there exists an isolated set $I$, in the complement of $B$, whose derived set $I^{\prime}$ is the boundary of $B$. It follows that corresponding to every closed set $B$ there is an analytic function whose singularities are precisely the points of $B$ and whose zeros have every point of the boundary of $B$ as a limit point.

The Mittag-Leffler theorem gives a complete characterization of the possible distribution of zeros of an analytic function, but it gives no information about the possible behavior of the function away from those zeros. The following theorem goes further, by preassigning not only the position of the zeros but something about the level curves as well.

THEOREM VII. Let $B$ be a given closed set and $I \subset C(B)$ be an isolated set such that $I^{\prime}$ is the boundary of $B$. Let $a_{1}, a_{2}, \cdots$ be the points of $I$, and $\gamma_{1}, \gamma_{2}, \cdots$ be a given sequence of mutually exterior circles, with the center of $\gamma_{i}$ at $a_{i}$, such that no point of $B$ is inside or on any $\gamma_{i}$. Then there exists a function $f(z)$ which is analytic except on $B$, which has a simple zero at each point of $I$, and whose level curve $C:|f(z)|=1$ is such that the part of $C$ which is inside $\gamma_{i}$ is a Jordan curve separating $a_{i}$ from $\gamma_{i}, i=1,2, \cdots$.

Here "inside $\gamma_{i}$ " is used in the sense of being on the same side of $\gamma_{i}$ as $a_{i}$.

Proof. Let $h(z)$ be any function with simple zeros at $a_{1}, a_{2}, \cdots$ and no other zeros, and analytic except on $B$. Let $M_{1}, M_{2}, \cdots$ be the greatest lower bounds of $|h(z)|$ on $\gamma_{1}, \gamma_{2}, \cdots$, respectively. Let $g(z)$ be a function (whose existence is asserted by the corollary to Theorem IV) which is analytic except on $B$ and which is such that $|g(z)|>1 / M_{i}$ on $\gamma_{i}$. Then the product $f(z)$ $=h(z) g(z)$ will be analytic inside and on $\gamma_{i}$ and $|f(z)|>1$ on $\gamma_{i}$. Hence there will be a part of the level curve $C$ which is inside $\gamma_{i}$ and which separates $a_{i}$ from $\gamma_{i}$. Thus $f(z)$ has the properties stated in the theorem.

Theorem VII is unsatisfactory in that it makes no assertion about the zeros of $f(z)$ other than those on $I$. 


\section{PART III}

11. Statement of the problem. A known expansion theorem $\left({ }^{6}\right)$ asserts that for any set of $n$ points $a_{1}, a_{2}, \cdots, a_{n}$ there exists a sequence of functions $\phi_{s}(z), s=1,2, \cdots$, satisfying the following two conditions:

(A) Each function $\phi_{s}(z)$ is analytic in a region including all the $a$ 's.

(B) Corresponding to any function $f(z)$ which is analytic at all the $a$ 's (but not necessarily analytic in any region containing more than one of the $a$ 's), there is a sequence of constants $c_{1}, c_{2}, \ldots$ such that

$$
f(z)=\sum_{s=1}^{\infty} c_{s} \phi_{s}(z) \text {. }
$$

This series converges absolutely and uniformly in some neighborhood of each point $a_{i}$.

The case of chief interest is that in which $f(z)$ cannot be continued analytically from $a_{i}$ to $a_{k}, i \neq k$. (For example, $f(z)$ might be $e^{z}$ near $a_{1}$, sin $z$ near $a_{2}, z^{2}$ near $a_{3}$, etc.)

We wish now to generalize this theorem by allowing $n$ to become infinite, so that $a_{1}, a_{2}, \cdots$ will form an arbitrary isolated set $I$. In doing this, certain characteristic differences with the finite case arise:

(1) The set of limit points $B$ of $I$ will be non-vacuous. One would expect the functions $\phi_{s}(z)$ to be badly behaved on $B$, particularly if $B$ divides the plane. We, therefore, replace condition (A) by the condition that $\phi_{s}(z)$ be analytic everywhere except on $B$.

(2) One might also expect that for a fixed set of functions $\left\{\phi_{8}(z)\right\}$ any corresponding $f(z)$ would of necessity satisfy certain uniformity conditions with respect to the points $a_{i}$. Such uniformity conditions are embodied in the following:

Definition. A function $f(z)$ will be said to belong to the class $G\left\{\theta_{i}, a_{i}\right\}$, where $\theta_{1}, \theta_{2}, \cdots$ is a sequence of positive numbers, provided $f(z)$ is analytic and uniformly bounded in a set of closed circular regions with centers at $a_{1}, a_{2}, \ldots$ and radii $\delta_{1}, \delta_{2}, \cdots$, where for some $\lambda$

$$
\delta_{i} \theta_{i} \geqq \lambda>0, \quad i=1,2, \cdots .
$$

We propose to prove the following theorem:

THEOREM VIII. Let $\left\{a_{i}\right\}$ be any isolated set of points with derived set $B$, and let $\left\{\theta_{i}\right\}$ be any sequence of positive numbers. Then there is a sequence of functions $\left\{\phi_{s}(z)\right\}$, each of which is analytic everywhere except on $B$, such that for any function $f(z)$ of class $G\left\{\theta_{i}, a_{i}\right\}$ there are numbers $c_{1}, c_{2}, \cdots$ for which

$$
f(z)=\sum_{s=1}^{\infty} c_{s} \phi_{s}(z)
$$

(6) Tôhoku Mathematical Journal, vol. 43 (1937), pp. 246-251. 
The series will converge absolutely and uniformly in some point set which contains a neighborhood of each point $a_{i}$.

For the above case in which $n$ is finite, the functions $\left\{\phi_{s}(z)\right\}$ depend only on the points $a_{1}, a_{2}, \cdots, a_{n}$, and not at all on $f(z)$. In Theorem VIII the functions $\left\{\phi_{s}(z)\right\}$ depend on $\theta_{1}, \theta_{2}, \cdots$ as well as upon the points $\left\{a_{i}\right\}$; but they are otherwise independent of $f(z)$.

The proof of Theorem VIII will be carried out in steps. It will be shown first that there exists a particular sequence of points $a_{1}, a_{2}, \cdots$ for which the theorem holds. A transformation will then be performed which will send this particular set of $a$ 's into an arbitrary sequence.

12. Proof of the expansion theorem for a particular sequence of points. The particular set of points $a_{1}, a_{2}, \cdots$ to be considered in this section will be a certain set of integers $k_{1}, k_{2}, \cdots$, where $k_{1}=0$ and $k_{i+1}>k_{i}$. It is desired to expand a function $f(z)$ of class $G\left\{\theta_{i}, k_{i}\right\}$. Let $\gamma_{1}, \gamma_{2}, \ldots$ be a sequence of mutually exterior circles with centers at $k_{1}, k_{2}, \cdots$; with radii $\beta_{1}, \beta_{2}, \cdots$, where the $\beta$ 's have a finite upper bound $\beta$, and $\beta_{i} \leqq 1 / \theta_{i}$; and in which $f(z)$ is analytic and uniformly bounded. According to Theorem VII there exists an integral function $\psi(z)$ which has a simple zero at each point $k_{i}$ and whose level curve $C_{\eta}:|\psi(z)|=\eta, \eta \leqq 1$, is such that the part of $C_{\eta}$ which is inside $\gamma_{i}$ is a Jordan curve separating $k_{i}$ from $\gamma_{i}$. Denote by $C_{\eta}^{(i)}$ this Jordan curve inside $\gamma_{i}$, and by $S_{\eta}^{(i)}$ the closed region containing $k_{i}$ which is bounded by $C_{\eta}^{(i)}$. Let $z_{1}$ be any point in $S_{\eta}^{(1)}$, and $z_{i}$ the point (there is one and just one) in $S_{\eta}^{(i)}$ where $\psi\left(z_{i}\right)=\psi\left(z_{1}\right)$.

Consider the infinite system of linear equations

$$
\sum_{j=1}^{\infty} g_{j}\left(z_{i}\right) h_{j}\left(z_{1}\right)=f\left(z_{i}\right), \quad i=1,2, \cdots,
$$

where the $g_{j}(z)$ are integral functions and the $h_{j}\left(z_{1}\right)$ are regarded as unknowns. We wish to define the $g_{j}(z)$ so that the determinant of the equations will be normal and different from zero $\left({ }^{7}\right)$. Let

$$
g_{j}(z)=e^{-\alpha_{j}\left(z-k_{j}\right)^{2}},
$$

where $\alpha_{1}, \alpha_{2}, \cdots$ are positive numbers such that $\sum \alpha_{j}$ converges. For the determinant $\Delta=\left|g_{j}\left(z_{i}\right)\right|$ to be normal it is sufficient that (a) the product of the diagonal terms converge absolutely and (b) the sum of the non-diagonal terms converge absolutely.

For (a) the product of the diagonal terms is

$$
\prod_{j=1}^{\infty} g_{j}\left(z_{j}\right)=\exp \left\{\sum_{j=1}^{\infty}-\alpha_{j}\left(z_{j}-k_{j}\right)^{2}\right\} .
$$

( $\left.{ }^{7}\right)$ For a discussion of normal determinants with applications to infinite systems of linear equations see F. Riesz, Les Systèmes d' Équations Linéaires, 1913, §§20-30. 
Since $\left|z_{j}-k_{j}\right| \leqq \beta_{j} \leqq \beta$, this product converges absolutely. The convergence is, moreover, uniform with respect to $z_{1}$ in $S_{\eta}^{(1)}$ and uniform for all sets of integers $\left\{k_{j}\right\}$. The value of the product is uniformly bounded away from zero for all $z_{1}$ in $S_{\eta}^{(1)}$ and all sequences of integers $\left\{k_{j}\right\}$.

For (b) we have the double series

$$
\sum_{i, j=1}^{\infty} g_{j}\left(z_{i}\right)=\sum_{i, j=1}^{\infty} \exp \left\{-\alpha_{j}\left(k_{i}-k_{j}+\lambda_{i}\right)^{2}\right\},
$$

where we have put $z_{i}=k_{i}+\lambda_{i},\left|\lambda_{i}\right| \leqq \beta_{i} \leqq \beta$. The prime indicates omission of terms for $i=j$.

Let $\epsilon$ be any positive number, and let $\left\{M_{i j}\right\}, i \neq j$, be any set of positive numbers such that $\sum M_{i j}=\epsilon$. We wish to show that the $k$ 's can be chosen so that the absolute value of the $i j$ th term of (5) will be less than $M_{i j}$.

We have

$$
\left|g_{j}\left(z_{i}\right)\right|<\exp \left\{-\alpha_{j}\left[\left(k_{i}-k_{j}\right)^{2}-2 \beta\left|k_{i}-k_{j}\right|-\beta^{2}\right]\right\} \equiv K_{i j} .
$$

The $k$ 's will now be chosen successively as follows: Let $k_{2}$ be so large that

$$
\max \left(K_{12}, K_{21}\right)<\min \left(M_{12}, M_{21}\right) .
$$

Then $k_{3}$ can be chosen so that

$$
\max _{j=1,2}\left(K_{j 3}, K_{3 j}\right)<\min _{j=1,2}\left(M_{j 3}, M_{3 j}\right) .
$$

In general, having already chosen $k_{2}, k_{3}, \cdots, k_{s-1}$, we then make $k_{s}$ so large that

$$
\max _{j<s}\left(K_{j 8}, K_{s j}\right)<\min _{j<s}\left(M_{j s}, M_{s j}\right) .
$$

Hence the terms of the series (5) are dominated by the numbers $M_{i j}$, where the latter may be preassigned arbitrarily. By proper choice of the $k$ 's the series will converge absolutely; hence $\Delta$ is normal. Moreover, the $k$ 's can be chosen so that the value of $\Delta$ differs from the value of the diagonal term by less than $\epsilon$. Hence, for suitable $k$ 's, $\Delta \neq 0$. From this point on, we assume that the $k$ 's are such that $\Delta$ is normal and nonvanishing.

Since $f(z)$ is bounded in the regions $S_{\eta}^{(i)}$ by a constant independent of $i$, the system of equations (4) will have one and just one solution $\left\{h_{s}\left(z_{1}\right)\right\}$ such that $\left|h_{s}\left(z_{1}\right)\right|$ is bounded for $z_{1}$ in $S_{\eta}^{(1)}$ by a constant independent of $s$. Since the convergence of the determinant is uniform, and since $f(z)$ is analytic in $S_{\eta}^{(1)}, S_{\eta}^{(2)}, \cdots$, then the functions $h_{s}\left(z_{1}\right)$ will each be analytic in $S_{\eta}^{(1)}$. Put

$$
h_{s}\left(z_{j}\right)=h_{s}\left(z_{1}\right) \text {. }
$$

Then $h_{s}(z)$ will be defined and analytic in each region $S_{\eta}^{(i)}$. Hence 


$$
f(z)=\sum_{j=1}^{\infty} e^{-\alpha_{j}\left(z-k_{j}\right)^{2}} h_{j}(z)
$$

for every $z$ in $S_{\eta}^{(1)}, S_{\eta}^{(2)}, \ldots$. The series on the right converges absolutely and uniformly on the same set of values of $z$.

Since $h_{j}(z)$ is analytic in $S_{\eta}^{(1)}$, it may be expanded in an absolutely and uniformly convergent series of powers of $\psi(z)$ :

$$
h_{j}(z)=\sum_{m=0}^{\infty} c_{j m}[\psi(z)]^{m} .
$$

But, since $h_{j}(z)$ and each term of its expansion is unchanged if $z_{1}$ is replaced by $z_{j}$, the expansion is equally valid for $z$ in $S_{\eta}^{(2)}, S_{\eta}^{(3)}, \ldots$. Hence

$$
f(z)=\sum_{j=1}^{\infty} e^{-\alpha_{j}\left(z-k_{j}\right)^{2}} \sum_{m=0}^{\infty} c_{j m}[\psi(z)]^{m}
$$

for $z$ in $S_{\eta}^{(1)}, S_{\eta}^{(2)}, \cdots$.

Let $M$ be the upper bound of $h_{j}(z)$ in $S_{\eta}^{(1)}$. Then, by an analogue of Cauchy's inequalities,

$$
\left|c_{j m}\right| \leqq M / \eta^{m}
$$

Hence, if $z$ is in $S_{\eta^{\prime}}^{(i)}, \eta^{\prime}<\eta$,

$$
\sum_{m=0}^{\infty}\left|c_{j m}[\psi(z)]^{m}\right| \leqq \sum_{m=0}^{\infty} \frac{M}{\eta^{m}} \eta^{\prime m}=\frac{M}{1-\eta^{\prime} / \eta} .
$$

We have already shown that $\sum g_{j}(z)$ converges absolutely. It follows that the double series in

$$
f(z)=\sum_{j=1, m=0}^{\infty} c_{j m} e^{-\alpha_{j}\left(z-k_{j}\right)^{2}}[\psi(z)]^{m}
$$

converges absolutely and uniformly to $f(z)$ in the regions $\left\{S_{\eta^{\prime}}^{(i)}\right\}$. Finally, $f(z)$ can be represented by any simple series that can be formed by rearrangement of the terms of this double series. This completes the proof of Theorem VIII in the special case of a particular function $f(z)$ of class $G\left\{\theta_{i}, k_{i}\right\}$. If $\bar{f}(z)$ is any other function of this same class, there will be a positive number $\lambda \leqq 1$ such that

$$
\delta_{i} \geqq \lambda / \theta_{i} \geqq \lambda \beta_{i} .
$$

Since $\psi(z)$ has a simple zero at $z=k_{i}$, it follows from Schwarz' lemma on lower bounds that $C_{\lambda \eta}^{(i)}$ will be inside the circle $\left|z-k_{i}\right|=\lambda \beta_{i}$. Hence $\bar{f}(z)$ will be analytic and uniformly bounded in the regions $S_{\lambda \eta}^{(i)}$ and will have an expansion in terms of the same set of functions as $f(z)$. 
13. Proof of the expansion theorem for an arbitrary isolated set. Consider any isolated set of points $\left\{a_{i}\right\}$ in an extended complex plane of the variable $x$. There exists a function $T(x)=z$ which is analytic except on $B$ and which has the values

$$
T\left(a_{i}\right)=k_{i}, \quad T^{\prime}\left(a_{i}\right)=\sigma_{i}, \quad i=1,2, \cdots,
$$

where $\sigma_{1}, \sigma_{2}, \cdots$ is a given sequence of complex numbers whose absolute values have a positive lower bound $\sigma$.

To construct such a function, we may first use the Weierstrass factor theorem to build a function $T_{1}(x)$ with a second order zero at each point $a_{1}, a_{2}, \cdots$. Let

$$
T_{1}(x)=\left(x-a_{i}\right)^{2}\left[\alpha_{i 1}+\alpha_{i 2}\left(x-a_{i}\right)+\cdots\right],
$$

where $\alpha_{i 1} \neq 0$. Next, by the Mittag-Leffler theorem, we form a function $T_{2}(x)$ with principal part at $a_{i}$ as follows:

$$
\frac{k_{i}}{\alpha_{i 1}} \frac{1}{\left(x-a_{i}\right)^{2}}+\frac{\sigma_{i} \alpha_{i 1}-k_{i} \alpha_{i 2}}{\alpha_{i 1}^{2}} \frac{1}{x-a_{i}} .
$$

Then the product $T(x)=T_{1}(x) T_{2}(x)$ will have the desired properties.

There exists an inverse function $x=T^{-1}(z)$ which is analytic at each point $k_{i}$, and

$$
T^{-1}\left(k_{i}\right)=a_{i}, \quad T^{-1}\left(k_{i}\right)=1 / \sigma_{i} .
$$

Hence, for every $\epsilon, 0<\epsilon<\sigma$, there exist positive numbers $\pi_{i}$ so small that in the regions $\left|z-k_{i}\right| \leqq \pi_{i}, T^{-1}(z)$ is analytic and

$$
\left|T^{-1}(z)-T^{-1}\left(k_{i}\right)\right| \leqq\left|z-k_{i}\right| /(\sigma-\epsilon) .
$$

We suppose that $f(x)$ is of class $G\left\{\tau_{i}, a_{i}\right\}$. Then $F(z) \equiv f\left(T^{-1}(z)\right)$ will be analytic and uniformly bounded if

$$
\left|z-k_{i}\right| \leqq \lambda(\sigma-\epsilon) / \tau_{i} \text { and } \leqq \pi_{i} \text {. }
$$

Hence, $F(z)$ will belong to the class $G\left\{\theta_{i}, k_{i}\right\}$ where $\theta_{i}=\max \left(\tau_{i}, 1 / \pi_{i}\right)$.

By the special case of Theorem VIII in the previous section, there exists a sequence of integral functions $\phi_{s}(z)$ such that any function of class $G\left\{\theta_{i}, k_{i}\right\}$ can be expanded in the form $F(z)=\sum c_{s} \phi_{s}(z)$ for some set of neighborhoods of $k_{1}, k_{2}, \cdots$. Hence $f(x)=\sum c_{s} \phi_{8}(T(x))$ for some set of neighborhoods of $a_{1}, a_{2}, \cdots$. The functions $\phi_{s}(T(z))$ will be analytic except for limit points of the $a$ 's and will depend only on the class $G\left\{\tau_{i}, a_{i}\right\}$ to which $f(x)$ belongs. This completes the proof of Theorem VIII.

UNIVERSITY OF ILLINOIS, URBANA, ILL. 